\title{
CALIBRATING PHOTOGRAMMETRIC AIRBORNE CAMERA SYSTEMS WITH DIFFRACTIVE OPTICAL ELEMENTS
}

\author{
D. Dahlke ${ }^{\mathrm{a}, *}$, M. Geßner ${ }^{\mathrm{a}}$, H. Meißner ${ }^{\mathrm{a}}$, K. Stebner ${ }^{\mathrm{a}}$, D. Grießbach ${ }^{\mathrm{a}}$, R. Berger ${ }^{\mathrm{a}}$, A. Börner ${ }^{\mathrm{a}}$ \\ ${ }^{\text {a }}$ Institute of Optical Sensor Systems, German Aerospace Center, 12489 Berlin, Germany - \\ (Dennis.Dahlke, Matthias.Gessner, Henry.Meissner, Karsten.Stebner, Denis.Griessbach, Ralf.Berger, Anko.Boerner)@ dlr.de
}

\author{
Commission I, WG 9
}

KEY WORDS: Diffractive Optical Elements, DOE, Geometric Calibration, Airborne, Test Field Calibration

\begin{abstract}
:
This paper presents a laboratory approach for geometric calibration of airborne camera systems. The setup uses an incoming laser beam, which is split by Diffractive Optical Elements (DOE) into a number of beams with precisely-known propagation directions. Each point of the diffraction pattern represents a point at infinity and is invariant against translation. A single image is sufficient to allow a complete camera calibration in accordance with classical camera calibration methods using the pinhole camera model and a distortion model. The presented method is time saving, since complex bundle adjustment procedures with several images are not necessary. It is well suited for the use with frame camera systems, but it works in principle also for pushbroom scanners. In order to prove the reliability, a conventional test field calibration is compared against the presented approach, showing that all estimated camera parameters are just insignificantly different. Furthermore a test flight over the Zeche Zollern reference target has been conducted. The aerotriangulation results shows that calibrating an airborne camera system with DOE is a feasible solution.
\end{abstract}

\section{INTRODUCTION}

The use of uncalibrated sensors to produce true-to-scale 3D models is increasing. Very often, almost true-to-scale results are sufficient for a wide range of applications. Nevertheless, this can also significantly increase the effort of image acquisition. Within the paper an innovative fast method for geometric aerial camera calibration by means of DOE in connection with laser beam equipment is presented. The use of metric aerial cameras requires precise knowledge about its interior orientation (IO). A conventional and common approach for geometric calibration of aerial cameras is the photogrammetric calibration using predefined calibration grids, rather huge 3D test fields or simultaneous on-the-job-calibration. Hereby ground truth data or ground control points (GCP) of the grid or 3D object are used to determine the relation between the camera coordinate system and the world reference frame. A sequence of images with several observations of the calibration target with different orientations are typically needed to estimate the camera parameters by minimizing a nonlinear error function.

In order to deploy a reliable modular airborne camera system for search and rescue tasks (MACS-SaR), the aforementioned procedures have been applied extensively in the past (Kraft et al., 2016). This is coherent with Honkavaara et al. (2008), who stated that the "calibration of the image acquisition system ... can only be performed under airborne conditions either using test fields or on a self-calibration basis...". However a much less complex and less time consuming calibration method is much more favoured when it comes to time critical tasks and repeated verification of IO parameters.

Laboratory setups have also been used in the past with single pixel illumination by collimated light. This calibration method uses collimator-goniometer arrangements to illuminate a set of

\footnotetext{
${ }^{*}$ Corresponding author
}

single pixels $(n \times m)$. Knowing the directions of the collimated light, it is possible to estimate the camera parameters (Schuster and Braunecker, 2000).

The calibration procedure reported here combines the particular advantages of calibration grid arrangements and single pixel illumination (Bauer et al., 2008). By using DOE as beam splitters only one image with $n \times m$ diffraction points is needed to estimate the interior camera parameters. An extended procedure is proposed here to calibrate an aerial camera system. This method has proven to work with terrestrial cameras and rather small focal lengths (Grießbach et al., 2009). Here it will be compared with a conventional photogrammetric test field approach as a reference. Furthermore both calibration results are applied to an aerotriangulation (AT) of a test flight over the Zeche Zollern reference target.

\section{CALIBRATION METHODS}

\subsection{Camera model}

For most camera calibration applications, the pinhole camera model (see Fig. 1) is assumed. In projective space $\mathbb{P}$ mapping of a homogeneous object point $\tilde{M} \in \mathbb{P}^{3}$ to an image point $\tilde{m} \in \mathbb{P}^{2}$ is defined with,

$$
\tilde{m}=P \tilde{M}
$$

where $\mathrm{P}$ is a $3 \times 4$-projection matrix consisting of the parameters of the interior- and exterior orientation of the camera.

$$
P=K[R \mid t]
$$

with $R, t$ describing the rotational matrix and translation of the exterior orientation relative to the world coordinate frame. The 


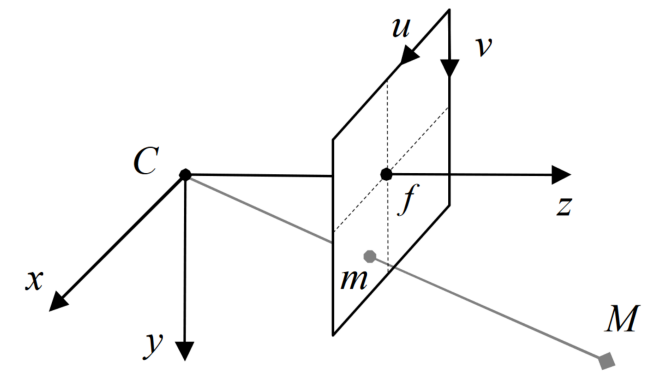

Figure 1. Pinhole camera model

camera matrix $K$ containing the focal length $f$ and the principal point $\left[u_{0}, v_{0}\right]^{T}$. The skew parameter is typically negligible in modern imaging devices and is therefore often set to zero.

$$
K=\left[\begin{array}{ccc}
f & 0 & u_{0} \\
0 & f & v_{0} \\
0 & 0 & 1
\end{array}\right]
$$

The ideal beam direction $\tilde{M}^{\prime}=\left[X^{\prime}, Y^{\prime}, Z^{\prime}\right]^{T}$ denoted in euclidean representation $\mathbb{R}^{3}$ is mapped into $2 \mathrm{D}$ image coordinates by projecting it on the plane $Z^{\prime}=1$

$$
\left[\begin{array}{l}
x \\
y \\
1
\end{array}\right]=\left[\begin{array}{l}
X^{\prime} / Z^{\prime} \\
Y^{\prime} / Z^{\prime} \\
1
\end{array}\right]
$$

with $x, y$ representing the ideal normalized image coordinates. From equation $(1,2)$ we get the ideal pixel image coordinates.

$$
\left[\begin{array}{l}
u \\
v \\
1
\end{array}\right]=K\left[\begin{array}{l}
x \\
y \\
1
\end{array}\right]
$$

Since the pinhole model assumes zero distortion in the image, a lens distortion model has to be considered before the pinhole model could be applied. Distortion models provide pixel by pixel correspondences between original images and their ideal undistorted images. Several distortion models are used in literature. Most commonly are models using rational functions (Claus and Fitzgibbon, 2005) or the radial distortion model by Brown (Brown, 1971). For comparison reasons we used the latter one for both presented approaches. Pincushion or barrel distortion is hereby expressed as follows,

$$
\left[\begin{array}{l}
\hat{x} \\
\hat{y}
\end{array}\right]=\left[\begin{array}{l}
x \\
y
\end{array}\right]\left(1+k_{1} r^{2}+k_{2} r^{4}+k_{3} r^{6}+\cdots\right)
$$

with

$$
r^{2}=x^{2}+y^{2}
$$

The complete mapping of ideal points to distorted image coordinates $[u, v]^{T}$ is subsumed to

$$
\left[\begin{array}{l}
x \\
y
\end{array}\right] \mapsto\left[\begin{array}{l}
\hat{u} \\
\hat{v}
\end{array}\right]=\left[\begin{array}{l}
u_{0} \\
v_{0}
\end{array}\right]+f\left[\begin{array}{l}
x \\
y
\end{array}\right]\left(1+k_{1} r^{2}+k_{2} r^{4}+k_{3} r^{6}+\cdots\right)
$$

Given a set of correspondent points $\tilde{M} \leftrightarrow[\hat{u}, \hat{v}]^{T}$ we seek to minimize the cost function

$$
\min _{m}\left\|\left[\begin{array}{c}
\hat{u}-u_{0} \\
\hat{v}-v_{0}
\end{array}\right]-f\left[\begin{array}{l}
x \\
y
\end{array}\right]\left(1+k_{1} r^{2}+k_{2} r^{4}+k_{3} r^{6}+\cdots\right)\right\|^{2}
$$

where $m=\left[f, u_{0}, v_{0}, k_{1}, k_{2}, k_{3}, \omega, \varphi, \kappa, \alpha, \beta\right]^{T}$ describing the interior and exterior orientation of the camera and a possible rotation $(\alpha, \beta)$ of the DOE in terms of the collimation coordinate frame. For the mapping to be invariant against translation, the exterior orientation only consists of the rotation matrix $R$ which is expressed by the Euler angles $\omega, \varphi, \kappa$. Since the test field calibration is not invariant against translation, $m$ in this case is set to $m=\left[f, u_{0}, v_{0}, k_{1}, k_{2}, k_{3}, \omega, \varphi, \kappa, X, Y, Z\right]^{T}$.

\subsection{Conventional test field calibration}

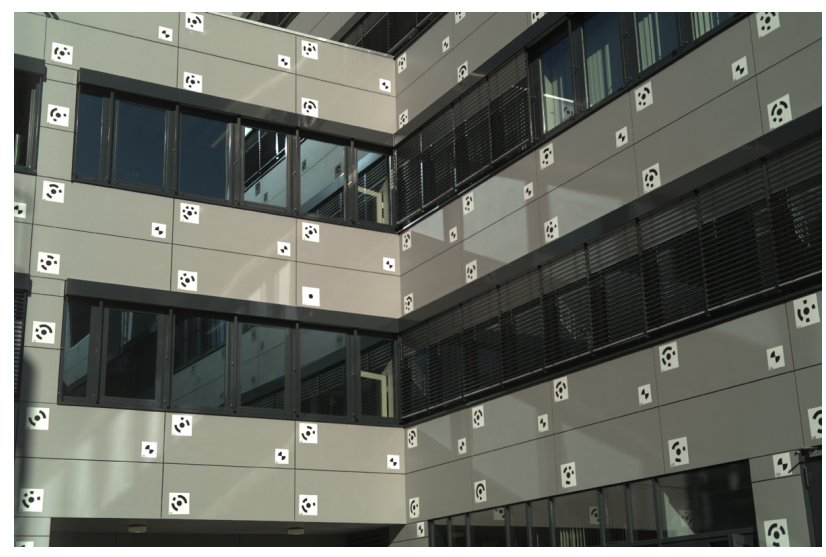

Figure 2. 3D Test Field at Beuth University of Applied Sciences Berlin

The presented calibration method is compared against a conventional photogrammetric approach using a three dimensional test field, which is located at Beuth University of Applied Sciences, Berlin. In the following the test field setup will be described in more detail to emphasize its complexity. It is equipped with coded markers, which are attached on two right-angled façades (see Figure 2). Each façade spans roughly 20 to $23 \mathrm{~m}$ across and 13 to $23 \mathrm{~m}$ in height. In order to serve as ground control points (GCPs), all markers have been measured with a tachymeter followed by a net adjustment resulting in a standard deviation of $1 \mathrm{~mm}$ in each dimension. The image acquisition was done at six locations. This guarantees different distances, line of sights and height levels in relation to the calibration target. Each observation was captured with different rotations $\left(0^{\circ}, 90^{\circ}, 180^{\circ}\right.$ and $\left.270^{\circ}\right)$ considering a homogeneous distribution of markers for each image. Rotations around the line of sight axis prevent high correlations between the principle point and the translation parameters of the exterior orientation (Luhmann et al., 2013). The point measurement was done automatically for each image due to the use of coded markers. All parameters for the interior and exterior orientation were derived during a bundle block adjustment.

\subsection{Camera calibration with Diffractive Optical Elements}

DOEs can be used to split an incoming laser beam with wavelength $\lambda$ into a number of beams with well-known propagation directions. As the image on the sensor is a Fraunhofer diffraction pattern, each projected image point represents a point at infinity, 
denoted in 3D projective space $\mathbb{P}^{3}$ by the homogeneous coordinate $\tilde{M}=[X, Y, Z, 0]^{T}$ with,

$$
\tilde{M}=\left[\begin{array}{c}
\lambda f_{x} \\
\lambda f_{y} \\
\sqrt{1-\lambda^{2}\left(f_{x}^{2}+f_{y}^{2}\right)} \\
0
\end{array}\right]
$$

where $f_{x}, f_{y}$ denote a spatial frequency encoded in the DOE. With suitable computational algorithms (Hermerschmidt et al., 2007) it is possible to encode spatially aperiodic DOEs with arbitrary spatial frequencies, choosing the propagation directions freely. As they are easier to design for the large aperture diameters needed spatially periodic DOEs were used here. Their spatial frequencies are given by $f_{x, y}=n_{x, y} / g_{x, y}$, with $n_{x}, n_{y}$ denoting the particular diffraction orders and grating constants $g_{x}, g_{y}$. The grating vectors are defining the $\mathrm{x}$ and $\mathrm{y}$ axis of the DOE coordinate frame. However, equation (10) is only valid if the incident light wave is a plane wave with uniform intensity distribution, perfectly perpendicular to the DOE surface. In a real setup, the beam is finite in extension and often has a non-uniform intensity profile, which is typically Gaussian. The deviations of the real beam profile from a plane wave cause the diffraction spots in the far field to have a certain size, which can be estimated from the Convolution theorem of Fourier Optics (Goodman, 2004). For a more detailed analysis, a laser beam can be expressed by its angular spectrum. The consequent propagation directions are determined with the diffraction formula for non-perpendicular incidence to the DOE, which needs to be applied in our analysis anyway because of the potentially unavoidable tilt of the DOE with respect to the incident laser beam. For the following analysis, the DOE coordinate system will be used, in which the incident beam is given by,

$$
r=[\sin (\beta),-\sin (\alpha) \cos (\beta), \cos (\alpha) \cos (\beta)]^{T}
$$

with the euler angles $\alpha$ and $\beta$ rotating the $x-$ and $y-$ axes of the DOE coordinate frame in terms of the collimator coordinate frame. The directions of the diffracted beams are now obtained as follows (McPhedran and Brown, 1980).

$$
\tilde{M}=\left[\begin{array}{c}
\lambda f_{x}+r_{x} \\
\lambda f_{y}+r_{y} \\
\sqrt{1-\left(\lambda f_{x}+r_{x}\right)^{2}-\left(\lambda f_{y}+r_{y}\right)^{2}} \\
0
\end{array}\right]
$$

It is straightforward to calculate the diffracted beam directions in the DOE coordinate frame by simple matrix operations, therefore the somewhat lengthy expressions that are obtained as a result will be omitted. In order to transform the beam directions into the camera coordinate frame, the exterior orientation of the camera in terms of the DOE coordinate frame has to be considered,

$$
\tilde{M}^{\prime}=\left[\begin{array}{cc}
R & t \\
0 & 1
\end{array}\right] \tilde{M}
$$

where $R$ is a $3 \times 3$ rotation matrix defining the camera orientation and $t$ the translation vector for the camera position. Equation (13) shows that the mapping of ideal points at infinity is invariant against translation which is a necessary condition for the following steps. This is also a great advantage compared to classical calibration grids for just one image is sufficient for calibration and therefore less parameters have to be estimated. The accuracy of the diffraction angles depends on the accuracy both of the wavelength and the grating constants, as can be seen from equation (10). Therefore, gas lasers emitting precisely given wavelengths in the visible were used, rather than diode lasers which can easily drift in wavelength. The angular accuracy was checked with a collimator-goniometer arrangement finding only minor deviations from the computed values of less than $0.001^{\circ}$.

Diffractive points are found by an iterative approach constantly refining the model parameters. The result is improved by calculating the centroids of the diffraction points which gives sub-pixel accuracy.

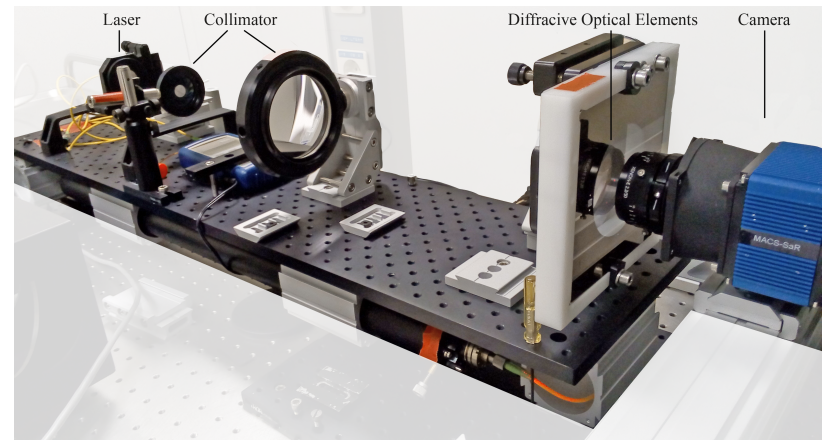

Figure 3. DOE Setup with the MACS Camera System

2.3.1 Single camera calibration setup The principle scheme for geometrical sensor calibration is illustrated in Figure 3. A helium-neon laser with a wavelength of $632.8 \mathrm{~nm}$ is collimated and enlarged with a beam expander to a diameter of $78 \mu \mathrm{m}$. The enlarged beam is then diffracted by a DOE which is located directly in front of the camera optics. The diameter of the incident laser beam and that of the DOE active area should be at least as large as the aperture diameter of the camera lens. Each of the diffracted beams is focused within the image plane of the camera. In order to obtain spots covering the whole sensor area, the maximum diffraction angle of the DOE should be larger than the field of view of the camera. No further alignment steps are necessary, because the mapping of the diffraction points is invariant against translation, and the rotation of the DOE in terms of the collimation system as well as the exterior orientation of the camera is modeled and can thus be determined (Bauer et al., 2008).

\section{RESULTS AND COMPARISON OF BOTH APPROACHES}

The numerical estimations of the camera parameters are performed on photogrammetric observations (image coordinates) and corresponding GCPs. In case of the DOE calibration, all image coordinates are derived from a single observation. Figure 4(a) shows the captured diffractive pattern. 1299 image points have been automatically detected with sub-pixel accuracy and were used for the subsequent calculation of the camera parameters. It can be seen that the distribution and spacing between illuminated points is very homogeneous over the whole sensor area. Thus the derived sensor model in general and the distortion model in particular should be applicable for the complete sensor. A very similar initial situation can be achieved with the test field calibration. Figure 4(b) shows the image coordinates and distribution of all detected GCPs. In this case 1529 image points have been automatically derived from 28 images observing 108 GCPs. 


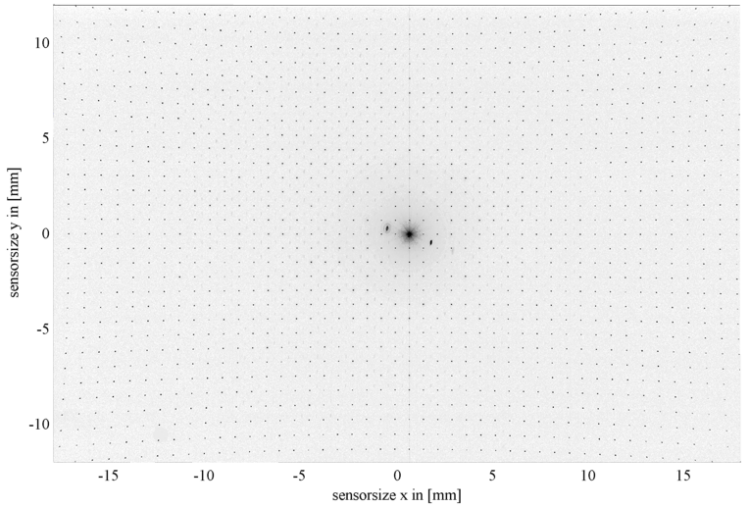

(a)

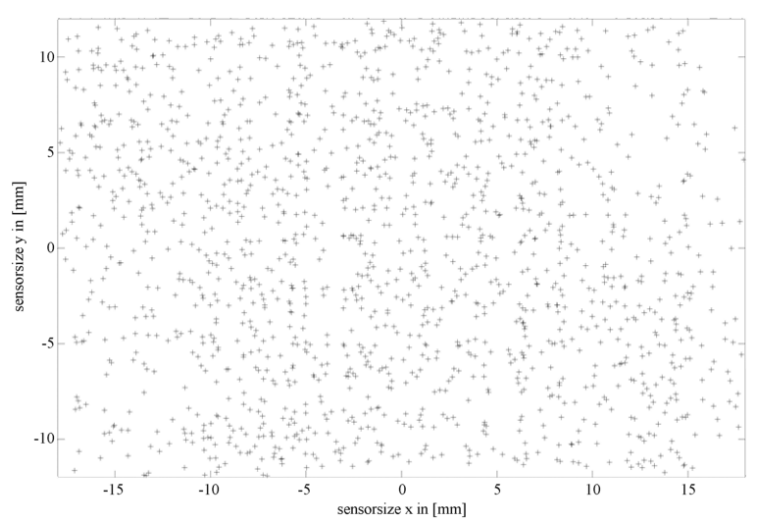

(b)

Figure 4. Sensor coverage with GCPs (a) Distribution of 1299 diffraction points (b) Distribution of 1529 GCP observations for the Beuth test field (sum of all images)

A summary of all derived intrinsic camera parameters can be found in Table 1. For both methods the $c_{k}$ parameter as well as the principle point is determined with an average accuracy of 0.25 pixel. Similarly the radial distortion parameters have been calculated significantly with roughly an order of magnitude below the $\mathrm{k}_{1}$ and $\mathrm{k}_{2}$ values. A closer look at the standard deviations (stdv) reveals an inverse behaviour between both approaches. On the one hand stdv of $c_{k}$ is almost three times higher than stdv for the principle point regarding the test field approach. On the other hand stdv of the principle point from DOE calibration is two times higher than $c_{k}$ stdv. However it can be stated, that the $c_{k}$ parameter as well as the principle point are significantly different for both approaches, since the distance between the parameters is larger than the sum of their corresponding stdv.

\begin{tabular}{|l|c|c|c|c|}
\hline & \multicolumn{2}{|c|}{ BHT test field } & \multicolumn{2}{c|}{ DOE } \\
\hline & value & stdv & value & stdv \\
\hline $\mathrm{c}_{\mathrm{k}}$ & $50.862 \mathrm{~mm}$ & $3.7 \mu \mathrm{m}$ & $50.851 \mathrm{~mm}$ & $1.5 \mu \mathrm{m}$ \\
\hline $\mathrm{x}_{0}$ & $-7.8 \mu \mathrm{m}$ & $1.1 \mu \mathrm{m}$ & $-17.9 \mu \mathrm{m}$ & $2.8 \mu \mathrm{m}$ \\
\hline $\mathrm{y}_{0}$ & $8.8 \mu \mathrm{m}$ & $1.4 \mu \mathrm{m}$ & $17.0 \mu \mathrm{m}$ & $2.7 \mu \mathrm{m}$ \\
\hline $\mathrm{k}_{1}$ & $1.81 \times 10^{-5}$ & $1.96 \times 10^{-7}$ & $1.99 \times 10^{-5}$ & $2.38 \times 10^{-6}$ \\
\hline $\mathrm{k}_{2}$ & $3.99 \times 10^{-9}$ & $4.31 \times 10^{-10}$ & $-1.01 \times 10^{-10}$ & $1.59 \times 10^{-11}$ \\
\hline
\end{tabular}

Table 1. Comparison of calibration results (Pixel Pitch of the camera is $7.4 \mu \mathrm{m}$ )

Radially symmetric distortions are plotted as a function of the image radius for both approaches in Figure 5(a). Because the deviation between both curves is almost not noticeable, the curve in Figure 5(b) depicts the difference between both. It can be seen that the correction differs by $3.4 \mu \mathrm{m}$ at most. However this large deviation, which corresponds to roughly 0.5 pixel, is only present between $60 \%$ and $80 \%$ of the image radius. Over almost half of the image radius the deviation is less than 0.2 pixel.

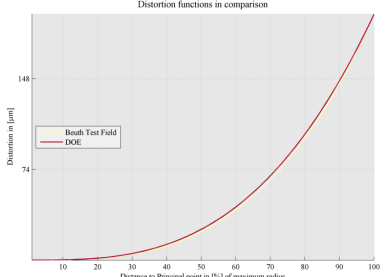

(a)

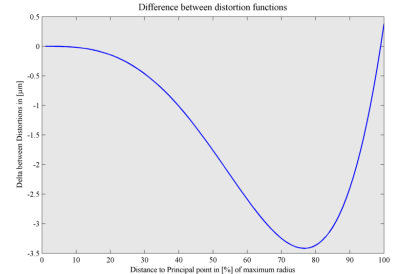

(b)
Figure 5. (a) Distortion functions from $\mathrm{k}_{1}$ and $\mathrm{k}_{2}$ for both approaches (b) DOE distortion function subtracted from test field distortion function

A negative correlation of roughly $95 \%$ is present between the two radial distortion terms for both approaches (see Figure 6(a) and Figure 6(b)). However it can also be seen that with the DOE calibration (Figure 6(a)) the $c_{k}$ remains correlated to both radial distortion parameters. With up to $90 \%$ this correlation is remarkably larger than its counterpart from the test field calibration (Figure 6(b)) with up to $20 \%$. All remaining correlations are similarly small for both approaches (less than $20 \%$ ).

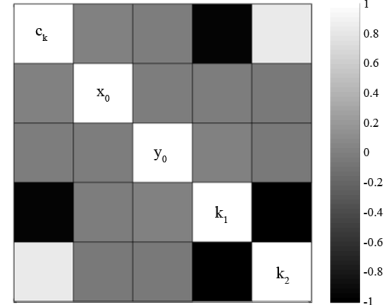

(a)

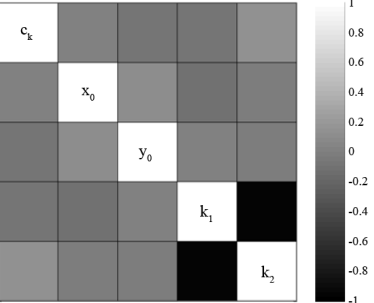

(b)
Figure 6. Covariances between intrinsic parameters. (a) Covariance plot for DOE calibration (b) Covariance plot for test field calibration

Investigations with DLR's MACS on a UAV at Zeche Zollern, a test field which is also used for ISPRS Benchmarks (Nex et al., 2015), have been conducted to assess the calibration accuracies of these different calibration methods. Altitude above ground level of the cross image flight was 95 and $110 \mathrm{~m}$ and results in a ground sampling distance of $1.4 \mathrm{~cm}$.

The area of the test field covers almost the entire area of the open space museum. Its extension is $320 \mathrm{~m} \times 220 \mathrm{~m}$. The highest vertical extent is given by the approx. $40 \mathrm{~m}$ high conveyor frames. It consists of 45 grid-shaped signaled ground points. The network measurements were carried out using tachymeters, a precision level and an RTK-GNSS system. The stdv of the adjusted heights are in a range of $1 \mathrm{~mm}$ to $3 \mathrm{~mm}$ and their relative accuracy is better than $1 \mathrm{~mm}$. For the network measurements a stdv of a single coordinate for the 15 common tachymeter survey stations, which could be measured in forced centering, was $1.2 \mathrm{~mm}$. The corresponding value for the datum points (GCP) was $2.5 \mathrm{~mm}$.

To control the IO parameter from both calibration methods, four ground control points in the corners and one in the center of the test field were used. The remaining 40 measuring points were 
used as checkpoints and the comparison of the respective results with ground truth. Both bundle adjustments using the same number of images, navigation data, corresponding image points, ground control points and the same weighting of input parameters. The focus of investigation was to find out whether the different but fixed IOs have an influence on the result with equally weighted input parameters. It can be shown that the difference of principle points tends to result in small systematic lateral shifts in an average range of GSD (see Figure 7). For images with less systematic shifts, increased angular diffenreces of up to $20 \mathrm{mdeg}$ are determined. The mean of height differences of adjusted EO parameter was about $4 \mathrm{~mm}$.

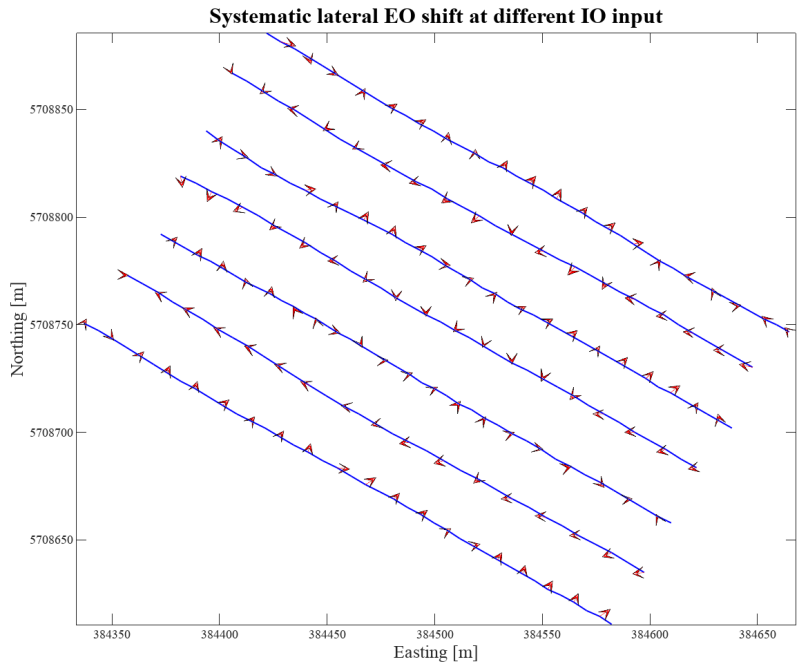

Figure 7. Direction of small systematic lateral differences (following the alternating flight pattern) of adjusted EO parameter

The RMS at 40 check points are comparable (see Figure 8).

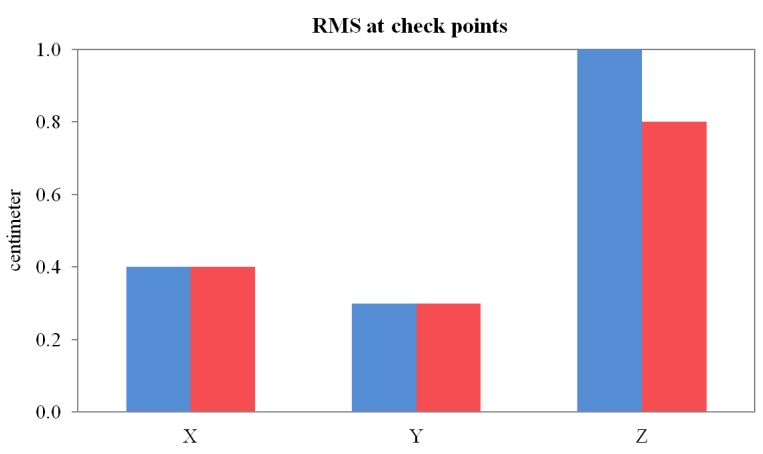

Figure 8. RMS at check points for BHT (blue) and DOE (red)

Figure 9 shows the absolute differences for all check points for both sets of IO parameter. The range and the direction of errors are almost identical with slightly increased differences in height accuracy.

Furthermore the cross image flight would have revealed inaccurate IO parameter with an increased RMS of image points. But this RMS respectively the reprojection error is as expected in range of 0.1 pixel for both bundle adjustments.
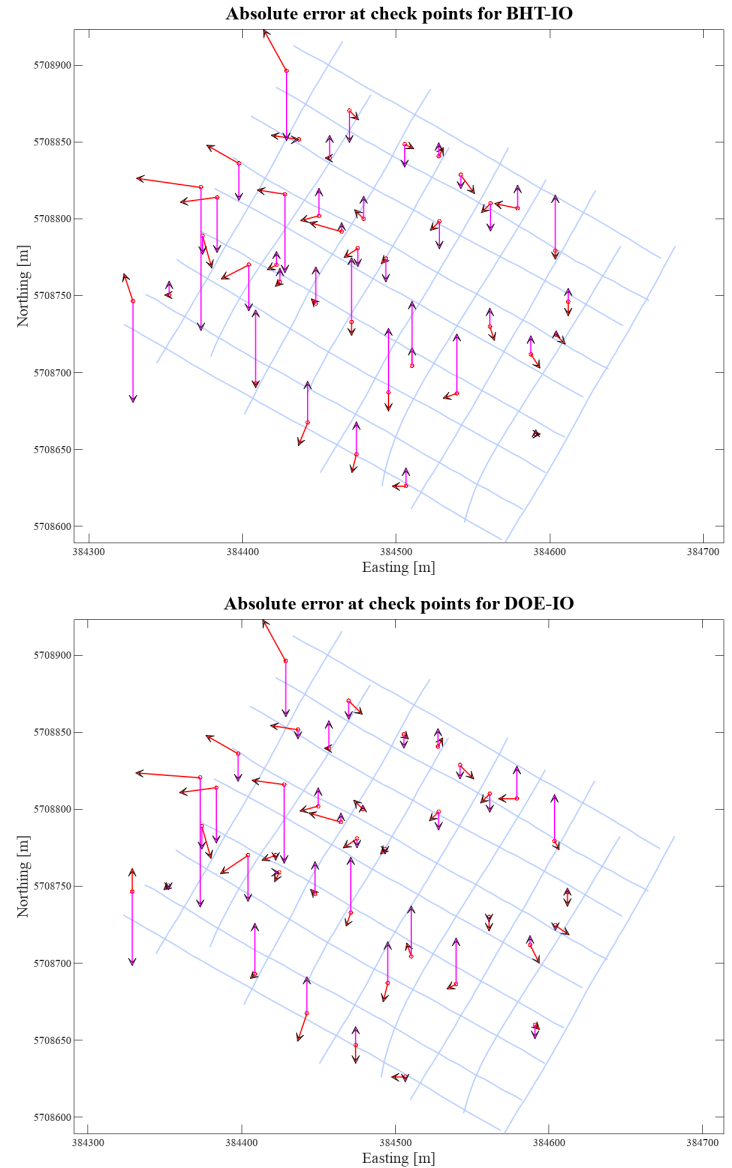

Figure 9. Absolute errors at check points with max error $2.5 \mathrm{~cm}$ (red horizontal, pink vertical, blue flight pattern)

\section{CONCLUSION AND OUTLOOK}

Concerning the presented aerial camera and the real world data set captured over Zeche Zollern, it is shown that a single image and a subsequent one-step calibration solution is sufficiently accurate to triangulate a cross image flight. Minimal differences of IO parameters end up in expectable minimal systematic differences of adjusted EO parameters. In principle, this has no impact on the final result in a corresponding procedure. Minor deviations in absolute height accuracy are not enough to favour a calibration method. Thus, the geometric calibration with DOE does not represent any significant disadvantages in the photogrammetric processing of the data, when compared to the common test field calibration. However it seems advantageous to have more observation with different orientations in order to decorrelate the focal length from the radial distortion parameters. Nevertheless it is an equivalent method and the determination of parameters of interior orientation is much more efficient and less prone to user errors.

Furthermore new investigation methods are rendered possible with this laboratory calibration setup and will be part of future work. Especially repeatability and long term stability of the described approach with diffractive optical elements will be explored using multi-temporal data sets over a sufficiently large time span. It has to be considered whether the results are reproducible for larger altitudes and other optical sensors. In addition our work will be focused on camera systems with large focal length and small field-of-view to clarify if the presented method (DOE) can be considered as a reliable calibration procedure. 


\section{ACKNOWLEDGEMENTS}

The authors of this paper would like to express their sincere gratitude to the employees of Labor für Photogrammetrie of the Beuth University of Applied Sciences for the possibility to use their test field. We greatly appreciate their effort to maintain such a reference target.

\section{REFERENCES}

Bauer, M., Grießbach, D., Hermerschmidt, A., Krüger, S., Scheele, M. and Schischmanow, A., 2008. Geometrical camera calibration with diffractive optical elements. Opt. Express 16(25), pp. 20241-20248.

Brown, D. C., 1971. Close-range camera calibration. Photogramm. Eng 37(8), pp. 855-866.

Claus, D. and Fitzgibbon, A. W., 2005. A rational function lens distortion model for general cameras. In: 2005 IEEE Computer Society Conference on Computer Vision and Pattern Recognition (CVPR'05), Vol. 1, pp. 213-219 vol. 1.

Goodman, J., 2004. Introduction to fourier optics 3rd edn (colorado: Roberts and company publishers).

Grießbach, D., Bauer, M., Hermerschmidt, A., Scheele, M. and Schischmanow, A., 2009. Stereo camera calibration with diffractive optical elements. In: 110. DGaO-Jahrestagung 2009.

Hermerschmidt, A., Krüger, S. and Wernicke, G., 2007. Binary diffractive beam splitters with arbitrary diffraction angles. $O p$ tics letters 32(5), pp. 448-450.

Honkavaara, E., Markelin, L., Ahokas, E., Kuittinen, R. and Peltoniemi, J., 2008. Calibrating digital photogrammetric airborne imaging systems in a test field. ISPRS - International Archives of the Photogrammetry, Remote Sensing and Spatial Information Sciences XXXVII-B1, pp. 555-560.

Kraft, T., Geßner, M., Meißner, H., Cramer, M., Przybilla, H.-J. and Gerke, M., 2016. Evaluation of a metric camera system tailored for high precision uav applications. In: International Society for Photogrammetry and Remote Sensing, Volume XLIB1, 2016.

Luhmann, T., Robson, S., Kyle, S. and Boehm, J., 2013. CloseRange Photogrammetry and $3 D$ Imaging. De Gruyter Textbook, De Gruyter.

McPhedran, R. and Brown, L., 1980. Electromagnetic theory of gratings. Springer Verlag, Berlin, pp. 227-275.

Nex, F., Gerke, M., Remondino, F., Przybilla, H.-J., Bäumker, M. and Zurhorst, A., 2015. Isprs benchmark for multi-platform photogrammetry. ISPRS Annals of Photogrammetry, Remote Sensing and Spatial Information Sciences II-3/W4, pp. 135142.

Schuster, R. and Braunecker, B., 2000. The calibration of the adc (airborne digital camera)-system. Int. Arch. of Photogrammetry and Remote Sensing pp. 288-294. 